\title{
A 52-year-old man with asymptomatic giant thoracic-abdominal aortic aneurysm
}

\author{
Jianying Deng ${ }^{1}$ and Wei Liu ${ }^{1}$ \\ ${ }^{1}$ Affiliation not available
}

March 28, 2021

\begin{abstract}
A 52-year-old man was admitted to our hospital for "CT-diagnosed thoracic-abdominal aortic aneurysm". One week ago, the patient had repeated dry coughs and went to the local hospital for treatment. A chest radiograph revealed a huge mass in the left thoracic cavity. A further chest CT examination revealed a thoracic-abdominal aortic aneurysm and was transferred to our hospital for surgical treatment.The patient is almost healthy, with no fever, no severe chest and abdomen pain, no dyspnea, no dysphagia or other clinical symptoms. Ten years ago, the patient underwent "ascending aorta and total aortic arch replacement surgery" in another cardiovascular hospital due to aortic dissection involving the ascending aorta and aortic arch (Debakey I). The patient's thoracic-abdominal aortic aneurysm is huge and has a high risk of rupture. Recently, the patient has undergone thoracic-abdominal aortic replacement surgery and is recovering well.
\end{abstract}

A 52-year-old man with asymptomatic giant thoracic-abdominal aortic aneurysm

Jianying Deng ${ }^{1}$, M.D. Wei $\mathrm{Liu}^{2}$, M.D.

${ }^{1}$ Department of Cardiovascular Surgery,Chongqing Kanghua Zhonglian Cardiovascular Hospital,

Chongqing 400015, China

${ }^{2}$ Department of Cardiac Surgery, DeltaHealth Hostital, Shanghai 201720, China

Correspondence: Jianying Deng, Department Of Cardiovascular Surgery,Chongqing Kanghua Zhonglian Cardiovascular Hospital, No. 168, Haier Road, District of Jiang Bei, Chongqing 400015, China. e-mail: 65673171@qq.com

Abstract A 52-year-old man was admitted to our hospital for "CT-diagnosed thoracic-abdominal aortic aneurysm". One week ago, the patient had repeated dry coughs and went to the local hospital for treatment. A chest radiograph revealed a huge mass in the left thoracic cavity. A further chest CT examination revealed a thoracic-abdominal aortic aneurysm and was transferred to our hospital for surgical treatment. The patient is almost healthy, with no fever, no severe chest and abdomen pain, no dyspnea, no dysphagia or other clinical symptoms. Ten years ago, the patient underwent "ascending aorta and total aortic arch replacement surgery" in another cardiovascular hospital due to aortic dissection involving the ascending aorta and aortic arch (Debakey I). The patient's thoracic-abdominal aortic aneurysm is huge and has a high risk of rupture. Recently, the patient has undergone thoracic-abdominal aortic replacement surgery and is recovering well.

Introduction

The patient was admitted to our hospital with no obvious clinical symptoms. At the time of admission, his vital signs were stable and his cough symptoms were basically cured. Blood pressure, 138/80 mmHg; heart rate, 79 beats/min; T, 36.5 ; respiratory rate, 16 breaths/min; arterial oxygen saturation on room air, $98 \%$. The height is $175 \mathrm{~cm}$, the weight is $85 \mathrm{~kg}$, and the body mass index(BMI) is $27.8 \mathrm{~kg} / \mathrm{m}^{2}$. There was no 
abnormal growth in the bones of the limbs. The patient is nearsighted and wears glasses to correct his vision in junior high school. The patient's laboratory test results are as follows: WBC, $6.2 \times 10^{\wedge} 9 / \mathrm{L}$; NEUT\%, $51 \%$; C-reactive protein, $7 \mathrm{mg} / \mathrm{L}$; procalcitonin, $0.20 \mathrm{ug} / \mathrm{L}$; total cholesterol, $6.1 \mathrm{mmol} / \mathrm{L}$; triglycerides, $2.99 \mathrm{mmol} / \mathrm{L}$; uric acid, 456umol/L. Tests for fungi and respiratory viruses were negative. The Covid-19 nucleic acid test was negative. Syphilis antibodies are negative.He has a history of hypertension with well controlled, and he has regular follow-up. He denied having hereditary diseases such as Marfan syndrome in his family, and denied his personal history of drug abuse and promiscuity. On the basis of these findings, a clinical diagnosis of non-Marfan syndrome of thoracic-abdominal aortic aneurysm was made.

\section{Discussion}

\section{Clinical Discussion}

Patients with thoracic-abdominal aortic aneurysms usually have no symptoms.Panneton and Hollier reported that about $43 \%$ of patients with thoracic-abdominal aortic aneurysms are asymptomatic, and most of these cases were discovered accidentally during unrelated imaging studies. ${ }^{1}$ The patient was normally healthy and had no symptoms of sudden chest pain. This time it was because of a "cough" that a chest CT examination was performed to find the thoracic-abdominal aortic aneurysm, which was an accidental discovery. For patients with aneurysm with no obvious clinical symptoms, especially those with a history of high-risk factors (such as hypertension, hyperlipidemia, Marfan syndrome and other family genetic diseases), we recommend regular follow-up visits to the hospital.

Although patients with thoracic-abdominal aortic aneurysms have no symptoms during the long-term course, there are still various clinical manifestations before the aneurysm is exception.Panneton and Hollier also reported that among $57 \%$ of symptomatic patients, $9 \%$ of patients had aneurysms ruptured. The most common symptom was back pain between the shoulder blades, while one of the main symptoms of other patients was compression. ${ }^{1}$ In this case, the patient's thoracic-abdominal aortic aneurysm is so giant that I have never seen before, and the maximum diameter of aneurysm is $144 \mathrm{~mm}$ (Fig.1 A-C), so the risk of aneurysm rupture is extremely high. We have adopted time-limited surgery to ensure the safety of the patient's life. During the operation, it was confirmed that the patient's thoracic-abdominal aortic aneurysm was huge, occupying most of the left thoracic cavity, and the operation was very difficult (Fig.2A). Fortunately, we succeeded in the operation (Fig.2B).

The causes of thoracic-abdominal aortic aneurysms include degeneration of the middle layer of the arterial wall (such as non-specific aortic middle layer degeneration and congenital connective tissue disease, such as Marfan syndrome, Ehlers-Danlos syndrome), aortic dissection, aortic arteritis, coarctation of the aorta, bacterial or fungal infection, etc., but degeneration of the middle layer of the arterial wall and aortic dissection are the main causes. ${ }^{2,3}$ In this case, Marfan syndrome, Ehlers-Danlos syndrome, congenital aortic aneurysm, and thoracic-abdominal aortic aneurysm caused by bacterial or fungal infections can be preliminarily ruled out from the characteristics of the medical history, physical examination, and laboratory examination. We are more inclined to consider non-specific aortic middle layer degeneration as the main cause of thoracoabdominal aortic aneurysm in this patient.

\section{Radiologic Discussion}

As a routine examination technique, chest radiograph still plays an important role in the initial examination and screening of aortic diseases. ${ }^{4}$ In this case, a chest radiograph for "cough" accidentally found a huge mass in the left thoracic cavity, prompting the physician to perform further chest CT examination to confirm the disease. Multi-slice CT angiography (CTA) can not only provide morphological information of luminal changes for aortic lesions, but also show more pathological conditions for vascular wall lesions (such as intra-aortic hematoma, aneurysm mural thrombus, aortic arteritis, etc.). Based on the advantages of CTA, such as fast, non-invasive, large field of view, higher temporal and spatial resolution, multiple reconstruction methods, and post-processing functions, CTA is more and more widely used in the treatment of aortic diseases diagnosis. ${ }^{5}$ This patient's chest and abdomen CTA (Fig.1A-C) revealed a true thoracic-abdominal aortic aneurysm, but not an aortic disscetion aneurysm. The thoracic-abdominal aortic aneurysm is extremely 
twisted and deformed; mural thrombus was seen in the aneurysm cavity, and multiple penetrating ulcers can be seen in the thoracic and abdominal aorta (Fig.1B); and the walls of the thoracic-abdominal aorta and bilateral common iliac arteries were calcified (Fig.1C). CTA also showed that the thoracic-abdominal aortic aneurysm was Crawford II. The proximal of the aneurysm starts at the distal of the artificial vessel, and the distal ended at bilateral common iliac arteries (Fig.1C).

Now, we routinely perform chest CT scans for patients with aortic disease, because chest CT can provide us with a lot of useful information. For this patient, based on the results of the CT scan, we made a decision for a time-limited surgery, and thoroughly discussed with the interventionalist, and finally decided to perform thoracic-abdominal aortic replacement surgery, and achieved satisfactory treatment results.

Pathologic Discussion

According to the extent of thoracic-abdominal aortic aneurysm expansion and involvement, Crawford divided the thoracic-abdominal aortic aneurysm into five types. This case was diagnosed as type II, that is, the lesion involves all the descending aorta and the abdominal aorta. From the results of the CT scan (Fig.1C) and the intraoperative resection of the aortic wall tissue (Fig.2B), the cause of the patient's thoracic-abdominal aortic aneurysm was almost arteriosclerosis. Histopathological results confirmed that the aneurysm wall was consistent with non-specific aortic wall degeneration, and genetic diseases such as Marfan syndrome were excluded.

Traditionally, we usually attribute the cause of aneurysms to arteriosclerosis. ${ }^{6}$ Clinically, aneurysms and arteriosclerosis are often co-exist, and the risk factors for the two are also similar. But in fact, the aneurysm is first caused by age-related non-specific aortic middle layer degeneration. ${ }^{7}$ Histologically, due to aging, the elastin in the middle layer of the aortic wall is broken, the collagen fibers are fibrillated, and the integrity and strength of the arterial wall are weakened. In addition, the hardened intima degrades the entire arterial wall, which in turn causes the aorta to expand or even rupture.

Conclusion

This patient was accidentally discovered a thoracic-abdominal aortic aneurysm and successfully underwent surgical treatment. We should thank the doctor who did chest radiograph and chest CT for saving the patient's life. For the subtle problems found in clinic, we should explore the reasons behind them. Some patients with thoracic-abdominal aortic aneurysms may not have typical clinical symptoms, but patients with high risk factors (such as hypertension, hyperlipidemia, etc.) should be followed up regularly in the hospital. Chest X-ray and CT scan are of great significance to aortic diseases. Thoracic-abdominal aortic replacement surgery is still the safest and most effective treatment for giant thoracic-abdominal aortic aneurysms.

Conflict of interest All authors declare that there is no conflict of interest.

Informed consent statemen t: Informed consent and ethical approval were waived for this report, which contains no patient identifiable data.

\section{References}

1. Panneton JM, Hollier LH. Nondissecting thoracoabdominal aortic aneurysms:Part I. Ann Vasc Surg.1995; 9(5):503-514.

2. Coselli JS, LeMaire SA, Miller 3rd CC, et al. Mortality and paraplegia after thoracoabdominal aortic aneurysm repair: a risk factor analysis. Ann Thorac Surg. 2000; 69(2): 409-414.

3. LeMaire SA, Carter SA, Volguina IV, et al. Spectrum of aortic operations in 300 patients with confirmed or suspected Marfan syndrome. Ann Thorac Surg. 2006,81(6):2063-2078.

4. Carpenter JP, Anderson WN, Brewster DC, et al. Multicenter pivotal trial results of the Lifepath System for endovascular aortic aneurysm repair. J Vasc Surg. 2004; 39(1):34-43.

5. Furukawa k, kamohara k., Nojiri J,et al. Operative strategy for descending and thoracoabdominal aneurysm repair with preoperative demonstration of the Adamkiewicz artery. Ann Thorac Surg. 2010; 90(6):1840-1846. 
6. DeBakey ME, Henly WS, Cooley DA, Morris Jr GC, Crawford ES, Beall Jr AC. Surgical management of dissecting aneurysms of the aorta. J Thorac Cardiovasc Surg, 1965;49:130-149.

7. Greenberg RK, Clair D, Srivastava S, et al. Should patients with challenging anatomy be offered endovascular aneurysm repair? J Vasc Surg.2003;38(5):990-996.

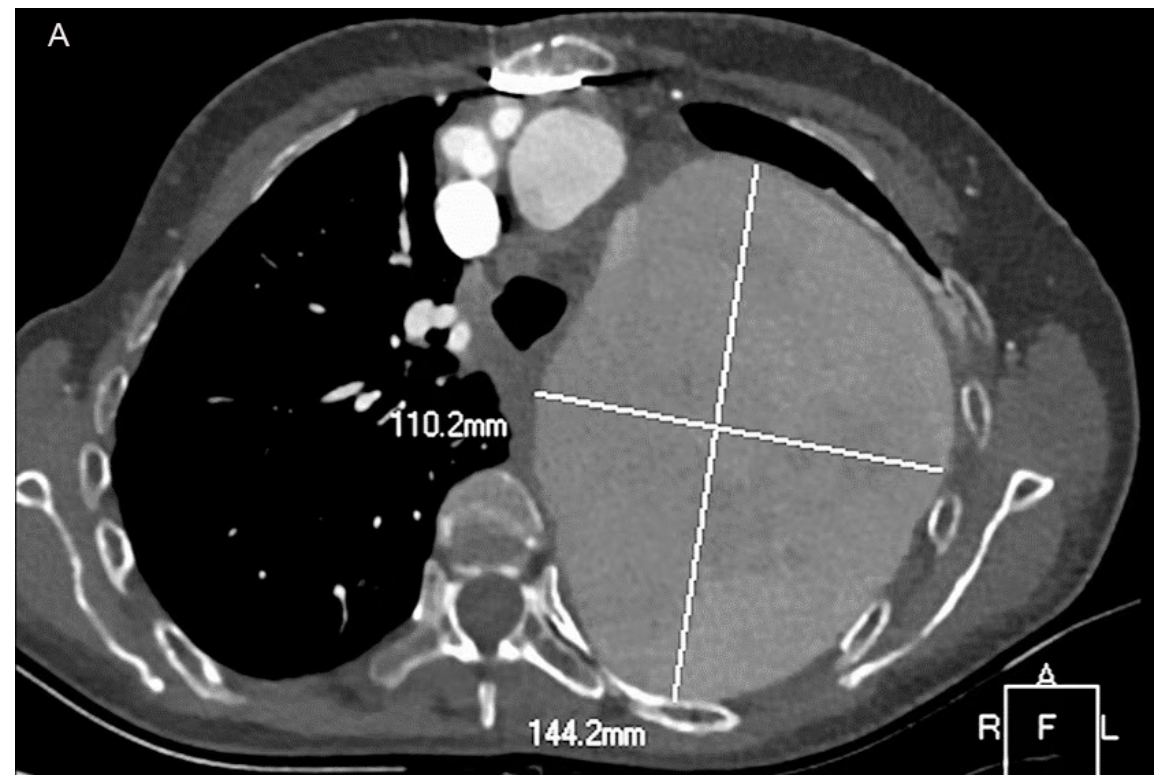



















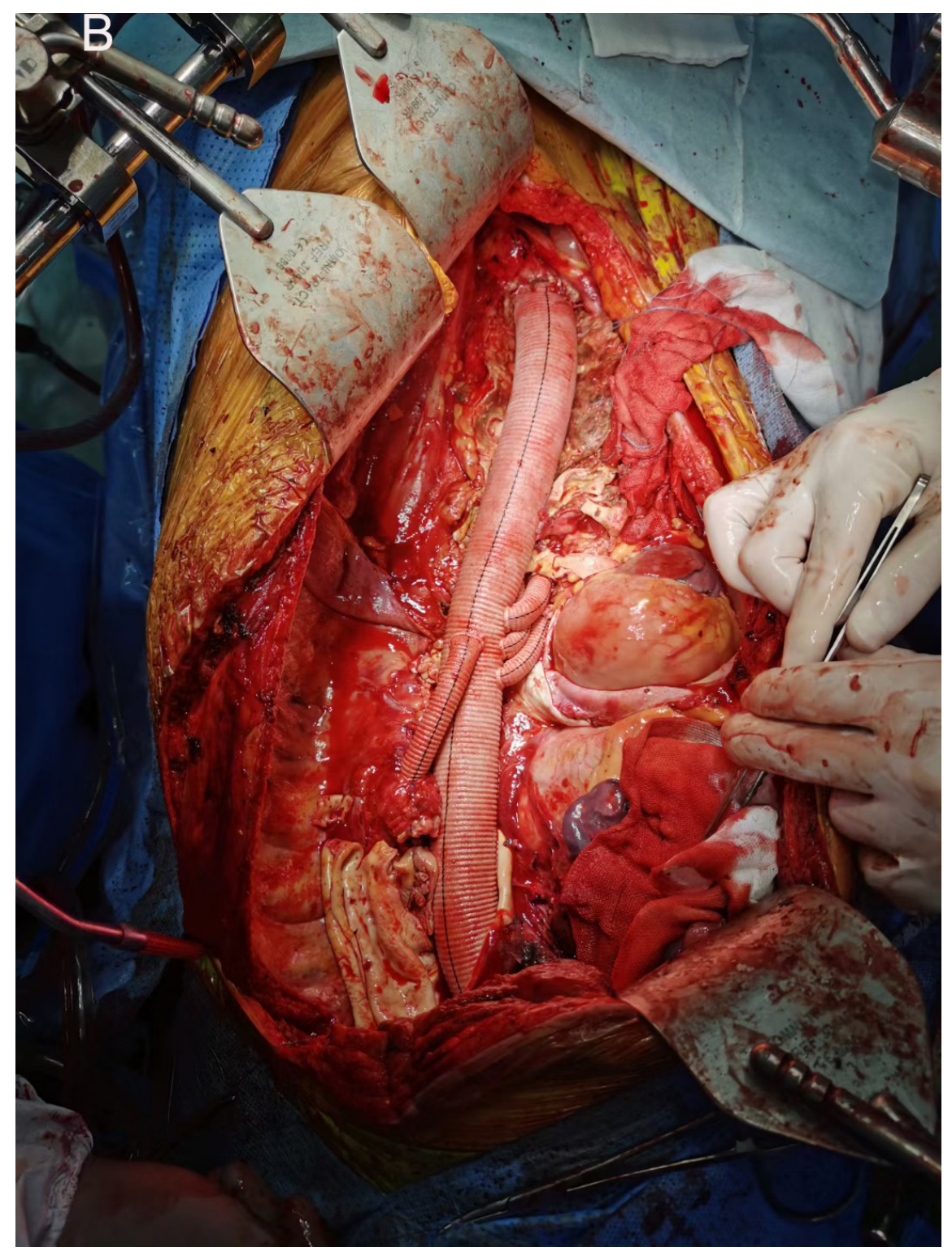

